\title{
Solvent-Free Synthesis of 5-Alkenyl-2,2-butylidene-1, 3-dioxane-4,6-diones under Ultrasonic Irradiation with o-Phthalimide-N-Sulfonic Acid as Catalyst
}

\author{
Chunhua Lin, Zhaohui Xu, Weilin Liao \\ Fine Chemical Key Laboratory of Jiangxi Province, Nanchang, China \\ Email: gotoxzh@163.om, liao1liao2@163.com
}

Received October 20, 2013; revised November 25, 2013; accepted December 11, 2013

Copyright (C) 2013 Chunhua Lin et al. This is an open access article distributed under the Creative Commons Attribution License, which permits unrestricted use, distribution, and reproduction in any medium, provided the original work is properly cited.

\begin{abstract}
5-Alkenyl-2,2-butylidene-1,3-dioxane-4,6-diones were synthesized by the Knoevenagel condensation reaction of aromatic aldehydes with 2,2-butylidene-1,3-dioxane-4,6-dione using o-phthalimide-N-sulfonic acid as catalyst, without solvent under ultrasonic irradiation. The present method has some notable advantages such as mild reaction conditions, short reaction times, less catalyst dosage and high yields with the green aspects by avoiding toxic catalysts and solvents. Further, the catalyst can be reused for five times without any noticeable decrease in the catalytic activity.
\end{abstract}

Keywords: o-Phthalimide-N-Sulfonicacid; 5-Alkenyl-2,2-butylidene-1,3-dioxane-4,6-diones; Aromatic Aldehydes; Knoevenagel Condensation

\section{Introduction}

In recent years, the preparation of 5-alkenyl-1,3-dioxane4,6-diones has attracted strong interest owing to the broad spectrum of their properties: these compounds can be employed as versatile intermediates for a series of natural products and heterocyclic compounds with potentially biological activity [1-5]. In addition, it is also reacted with Grignard reagent in conjugate addition [6] or conjugated dienes in Diels-Alder cycloaddition [7]. In particular, 5-alkenyl-2,2-dimethyl-1,3-dioxane-4,6-dione is an important reagent for synthesizing lipid-lowering drugs "Lipitor" [8]. Therefore, the preparation of 5-alkenyl-1,3-dioxane-4,6-diones is of much current importance.

More recently, lots of methods for the synthesis of 5alkenyl-1,3-dioxane-4,6-diones have been reported in the literature. Generally, they were synthesized by the Knoevenagel condensation reaction of 1,3-dioxane-4,6diones and aromatic aldehydes in the presence of bases such as pyridine $[9,10]$, piperidine/glacial acetic acid [11, 12], or hexamethyldisilazane [13] and Lewis acids, such as anhydrous zinc chloride [14], $\mathrm{TiCl}_{4} /$ pyridine [15] and $\mathrm{CePW}_{12} \mathrm{O}_{40}$ [16]. However, many of these methodologies have not been entirely satisfactory, owing to such drawbacks as low yields, long reaction time, more catalyst dosage, environmentally unfavorable solvents, emerging the problems of tedious work-up and effluent pollution. Uncatalyzed reaction was also reported in the literature using DMF or DMSO as solvent, which is toxic, teratogenic and suspected carcinogen. Those methods gave mixtures of unsaturated and Michael addition products [17]. Thus, a mild, efficient, and environmentally friendly method using economical catalyst is desirable.

Recently, the solvent-free condition and the use of heterogeneous catalysts have emerged as an eco-friendly alternative of great importance within organic synthesis, as they reduce environmental pollution and bring down handling costs due to simplification of work-up technique [18].

Ultrasound has increasingly been used in organic synthesis in the last three decades. Compared with traditional methods, the procedure is more convenient and can be carried out in higher yields, shorter reaction time or milder conditions under ultrasound irradiation [19-23]. Our investigations on the application of ultrasound in organic synthesis [16] and our work on Knoevengel condensations $[13,24]$ are continued. Herein, we would like to report an efficient and practical procedure for the synthesis of 5-alkenyl-2,2-butylidene-1,3-dioxane-4,6-diones with 2,2-butylidene-1,3-dioxane-4,6-dione [23,25] and 
aromatic aldehydes in o-phthalimide- $\mathrm{N}$-sulfonic acid (OPNSA) without solvent under ultrasound irradiation (Scheme 1).

\section{Results and Discussion}

To optimize the reaction conditions, a selected model reaction was carried out with 2,2-butylidene-1,3-dioxane4,6-dione 1 ( $0.01 \mathrm{~mol}, 1.7 \mathrm{~g})$, benzaldehyde 2 (0.01 mol) and different sets of reaction conditions. The results are summarized in Table 1. The results clearly show that the catalyst is essential, the catalytic activity of o-phthalimide-N-sulfonic acid (OPNSA) is high, giving 3a in high yield in a short reaction time. Using OPNSA as the catalyst, we evaluated the reaction in various solvents and under solvent-free conditions. The product yield in a polar solvent was higher than the one in non-polar solvent, but under solvent-free conditions the highest yield was obtained (Table 1, Entries 1-8). The catalyst (OPNSA) plays a crucial role in the success of the reaction in terms of the yields. The presence of $0 \mathrm{~mol} \%$ OPNSA gave the product 3a in quantitative yield $(35 \%)$ at $60^{\circ} \mathrm{C}$. Increasing the catalyst to 1,3 , and $5 \mathrm{~mol} \%$ results in improved reaction yields to $76 \%, 89 \%$ and $87 \%$ respectively (Table 1, Entries 8-11). Use of just $3 \mathrm{~mol} \%$ OPNSA is sufficient to push the reaction forward. Higher amounts of the catalyst did not improve the results to a great extent. Thus, $3 \mathrm{~mol} \%$ OPNSA was chosen as a quantitative catalyst for these reactions.

To find the optimum reaction time, the reaction was carried out in the presence of OPNSA for 20, 30, 40 and 60 minutes, resulting in the isolation of $3 a$ in $90 \%, 91 \%$, $90 \%$ and $89 \%$ yield, respectively. Similarly, the optimum reaction temperature was $30^{\circ} \mathrm{C}$. In addition, it must be pointed out that all of these reactions were carried out without solvent under ultrasonic irradiation. Under mechanical agitation conditions the product yield for $68 \%$ and with ultrasonic irradiation the product yield for $91 \%$, which indicated the ultrasonic radiation to promote the reaction effectively (Table 1, Entries 16, 17). The best results was obtained when was conducted at $30^{\circ} \mathrm{C}$, for 30 minutes in the presence of $3 \mathrm{~mol} \%$ OPNSA (Table 1,

\section{Entry 16).}

To determine the generality of this method, the scope of the reaction was investigated using a number of aromatic aldehydes under the optimized reaction conditions. The results are presented in Table 2. The results show benzaldehyde and aromatic aldehydes with electron with drawing groups $\left(-\mathrm{Cl},-\mathrm{NO}_{2},-\mathrm{F}\right)$ and electron-donating groups $\left(-\mathrm{OCH}_{3},-\mathrm{OH},-\mathrm{N}\left(\mathrm{CH}_{3}\right)_{2}\right)$ have all provided high yields of the products.

The principle advantages of the use of o-phthalimide$\mathrm{N}$-sulfonic acid (OPNSA) in organic transformations are their reusability. The catalyst was readily recovered from
Table 1. Optimization of reaction conditions for synthesizing compound (3a) ${ }^{\mathrm{a}}$.

\begin{tabular}{|c|c|c|c|c|c|c|}
\hline Entry & Solvent & $\begin{array}{l}\text { Catalyst } \\
(\mathrm{mol} \%)\end{array}$ & $\begin{array}{c}\text { Temperature } \\
\left({ }^{\circ} \mathrm{C}\right)\end{array}$ & $\begin{array}{l}\text { Time } \\
(\mathrm{min})\end{array}$ & $\begin{array}{l}\text { Yield }^{\mathrm{b}} \\
(\%)\end{array}$ & $\begin{array}{c}\text { Experiment } \\
\text { method }\end{array}$ \\
\hline 1 & $\mathrm{C}_{2} \mathrm{H}_{5} \mathrm{OH}$ & 3 & Reflux & 120 & 81 & Ultrasonic \\
\hline 2 & $\mathrm{CH}_{3} \mathrm{OH}$ & 3 & Reflux & 120 & 80 & Ultrasonic \\
\hline 3 & $\mathrm{CH}_{3} \mathrm{CN}$ & 3 & Reflux & 150 & 72 & Ultrasonic \\
\hline 4 & THF & 3 & Reflux & 150 & 73 & Ultrasonic \\
\hline 5 & $\mathrm{C}_{6} \mathrm{H}_{6}$ & 3 & Reflux & 180 & 66 & Ultrasonic \\
\hline 6 & $\mathrm{CH}_{2} \mathrm{Cl}_{2}$ & 3 & Reflux & 210 & 68 & Ultrasonic \\
\hline 7 & $\mathrm{H}_{2} \mathrm{O}$ & 3 & Reflux & 120 & 78 & Ultrasonic \\
\hline 8 & None & 3 & 60 & 60 & 89 & Ultrasonic \\
\hline 9 & None & 5 & 60 & 60 & 87 & Ultrasonic \\
\hline 10 & None & 1 & 60 & 60 & 76 & Ultrasonic \\
\hline 11 & None & 0 & 60 & 120 & 35 & Ultrasonic \\
\hline 12 & None & 0 & 60 & 120 & 24 & Mechanical \\
\hline 13 & None & 3 & 50 & 60 & 89 & Ultrasonic \\
\hline 14 & None & 3 & 40 & 40 & 90 & Ultrasonic \\
\hline 15 & None & 3 & 30 & 30 & 91 & Ultrasonic \\
\hline 16 & None & 3 & 30 & 20 & 90 & Ultrasonic \\
\hline 17 & None & 3 & 30 & 60 & 68 & Mechanical \\
\hline
\end{tabular}

${ }^{\text {a }}$ Reaction conditions: benzaldhyde $(0.01 \mathrm{~mol}), 2$,2-butylidene-1,3-dioxane4,6-dione $(1.70 \mathrm{~g}, 0.01 \mathrm{~mol})$, solvent $(10 \mathrm{~mL})$ or solvent-free conditions (power ultrasonic irradiation $250 \mathrm{~W}$, irradiation frequency $40 \mathrm{kHz}$ ); ${ }^{\mathrm{b}}$ Isolated yields.

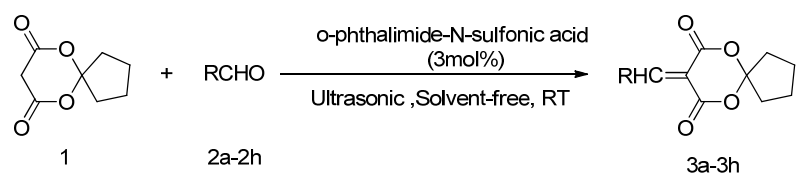

Scheme 1. Synthesis of 5-alkenyl-2,2-butylidene-1,3-dioxane-4,6-diones.

the reaction mixture using the procedure outlined in the experimental section. The separated catalyst was washed with 2-methoxy-2-methylpropane $(10 \mathrm{~mL})$, it was directly used in a similar reaction. From Table 3 we found that the catalyst could be used at least five times with only a slight reduction in activity $(91 \%$ yield for first use, $91 \%$ for second use, $90 \%$ for third use, $89 \%$ for fourth time and $86 \%$ for fifth time).

A plausible mechanism for the formation of the 5-alkenyl-2,2-butylidene-1,3-dioxane-4,6-diones products using o-phthalimide-N-sulfonic acid (OPNSA) as a catalyst has been depicted in Scheme 2. 
Table 2. OPNSA-catalyzed synthesis of 5-alkenyl-2,2-butylidene-1,3-dioxane-4,6-diones (3a-3h) ${ }^{\mathrm{a}}$.

\begin{tabular}{|c|c|c|c|c|c|}
\hline \multirow[b]{2}{*}{$\mathrm{R}$} & \multirow{2}{*}{ Product } & \multirow{2}{*}{$\begin{array}{l}\text { Time } \\
(\mathrm{min})\end{array}$} & \multirow{2}{*}{$\begin{array}{c}\text { Yield }^{\mathrm{b}} \\
(\%)\end{array}$} & \multicolumn{2}{|c|}{ Melting point $\left({ }^{\circ} \mathrm{C}\right)$} \\
\hline & & & & Found & Reported [Ref.] \\
\hline $\mathrm{C}_{6} \mathrm{H}_{5}$ & $3 a$ & 30 & 91 & $87-88$ & $88-90$ [26] \\
\hline 4-HO- $\mathrm{C}_{6} \mathrm{H}_{4}$ & $3 b$ & 35 & 88 & $170-172$ & $170-172[26]$ \\
\hline 4- $\mathrm{CH}_{3} \mathrm{O}-\mathrm{C}_{6} \mathrm{H}_{4}$ & $3 \mathrm{c}$ & 35 & 89 & $83-85$ & $83-85$ [16] \\
\hline $4-\mathrm{NO}_{2}-\mathrm{C}_{6} \mathrm{H}_{4}$ & $3 \mathrm{~d}$ & 15 & 93 & $150-152$ & $150-152[16]$ \\
\hline $2-\mathrm{NO}_{2}-\mathrm{C}_{6} \mathrm{H}_{4}$ & $3 e$ & 20 & 91 & $112-114$ & $113-115[16]$ \\
\hline 4-F-C ${ }_{6} \mathrm{H}_{4}$ & $3 \mathrm{f}$ & 15 & 87 & $86-88$ & $86-88$ [26] \\
\hline $4-\mathrm{Cl}-\mathrm{C}_{6} \mathrm{H}_{4}$ & $3 g$ & 20 & 84 & $115-116$ & $114-116[16]$ \\
\hline $4-\left(\mathrm{CH}_{3}\right)_{2} \mathrm{~N}-\mathrm{C}_{6} \mathrm{H}_{4}$ & $3 \mathrm{~h}$ & 25 & 92 & $180-182$ & $180.5-181[16]$ \\
\hline
\end{tabular}

${ }^{\mathrm{a}}$ Reaction conditions: benzaldhyde $(0.01 \mathrm{~mol}), 2$,2-butylidene-1,3-dioxane-4, 6-dione $(1.70 \mathrm{~g}, 0.01 \mathrm{~mol})$, OPNSA catalyst $(3 \mathrm{~mol} \%), 30^{\circ} \mathrm{C}$, solvent-free conditions (power ultrasonic irradiation $250 \mathrm{~W}$, irradiation frequency 40 $\mathrm{kHz})$; ${ }^{\mathrm{b}}$ Isolated yields.

Table 3. The recycling of OPNSA in synthesis of 3a under the reaction optimized conditions.

\begin{tabular}{ccc}
\hline Entry & Time $(\mathrm{min})$ & Yield $^{\mathrm{a}}(\%)$ \\
\hline 1 & 30 & 91 \\
2 & 35 & 91 \\
3 & 35 & 90 \\
4 & 38 & 89 \\
5 & 40 & 86 \\
\hline
\end{tabular}

${ }^{\mathrm{a}}$ Isolated yields.
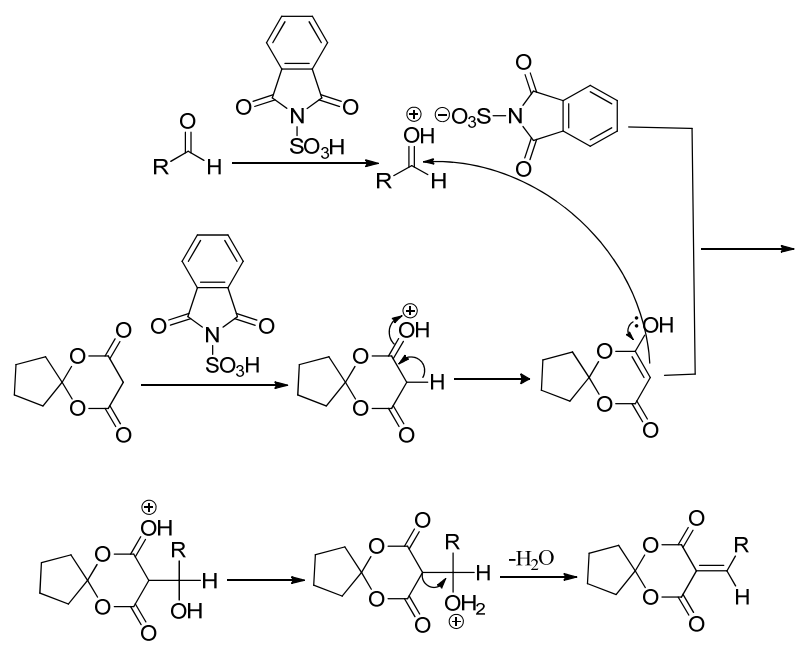

Scheme 2. The plausible mechanism for synthesis of the 5alkenyl-2,2-butylidene-1,3-dioxane-4,6-diones.

\section{Conclusion}

The catalyst of o-phthalimide-N-sulfonic acid (OPNSA), showed high catalytic activity in the synthesis of 5-alkenyl-2,2-butylidene-1,3-dioxane-4,6-diones synthesized by the Knoevenagel condensation reaction of aromatic aldehydes with 2,2-butylidene-1,3-dioxane-4,6-dione without solvent under ultrasonic irradiation. This procedure offers several advantages over the other techniques available in the literature, including much shorter reaction times, higher yields, milder conditions, and the absence of any hazardous organic solvents, which makes it a useful and attractive protocol for the synthesis of these compounds. Furthermore, the catalyst could be recycled after a simple work-up, and used at least five times without significant reduction in its catalytic activity.

\section{Experimental Section}

\subsection{Reagents and Instruments}

All chemicals were purchased from Aladdin, Aldrich and Fluka Chemical Companies and without further purification. The melting points of the various compounds were measured by XT-4 digital micro melting point instrument and are uncorrected. Reaction monitoring was accompanied by TLC using silica gelSIL G/UV 254 plates. KQ250E-machine ultrasonic instrument is made in Kunshan Co., LTD. IR spectra were taken on a Nicolet-360 FT-IR spectrometer by incorporating samples in $\mathrm{KBr}$ disks. ${ }^{1}$ HNMR spectra were recorded with Bruker Avance 400 $\mathrm{MHz}$ spectrometer with $\mathrm{CDCl}_{3}$ as the solvent and TMS as the internal standard. The ${ }^{13} \mathrm{CNMR}$ data were collected on Bruker Avance $100 \mathrm{MHz}$ instrument with $\mathrm{CDCl}_{3}$ as the solvent and TMS as the internal standard.

\subsection{Preparation of o-Phthalimide-N-Sulfonic Acid (OPNSA)}

A solution of chlorosulfonic acid $(11.6 \mathrm{~g}, 0.1 \mathrm{~mol})$ in $\mathrm{CH}_{2} \mathrm{Cl}_{2}(20 \mathrm{ml})$ was added to o-Phthalimide $(14.7 \mathrm{~g}, 0.1$ mol $)$ in $\mathrm{CH}_{2} \mathrm{Cl}_{2}(20 \mathrm{ml})$ solution at $0^{\circ} \mathrm{C}$. Then the mixture was stirred at room temperature for $24 \mathrm{~h}$. The solvent was evaporated at reduced pressure and the remaining solid was washed with 2-methoxy-2-methylpropane $(3 \times 10 \mathrm{~mL})$ and filtered to give the desired product as a yellow solid material in $97 \%$ yield.

\subsection{General Procedure for Synthesis of 5-Alkenyl-2,2-butylidene-1,3-dioxane-4, 6-diones}

2,2-butylidene-1,3-dioxane-4,6-dione 1 (0.01 mol, $1.7 \mathrm{~g}$ ), aromatic aldehyde 2 (2a-2h, $0.01 \mathrm{~mol})$ and o-phthalimide-N-sulfonic acid (OPNSA) $3 \mathrm{~mol} \%$ were taken in a round bottom flask, then the mixture was stirred under ultrasound irradiation at room temperature for $15-35$ min. Upon completion of the reaction, as confirmed by thin-layer chromatography (petroleum ether/ethyl acetate 
$5: 1$, the reaction mixture was diluted with $20 \mathrm{~mL}$ ethanol. The catalyst was collected from the filtrate, which was concentrated on reduced pressure. After the remaining catalyst was washed with 2-methoxy-2-methylpropane $(10 \mathrm{~mL})$, it was directly used for the next reaction. The crude solid product was filtered and then purified by recrytallization from ethanol to afford the pure product 3 (3a-3h). The physical data (mp, IR, NMR) of known compounds were identical to the corresponding literature data.

\subsection{The Data for Representative Compounds}

5-Benzylidene-2,2-butylidene-1,3-dioxane-4,6-dione (Compound 3a, Table 1): White solid (yield: 91\%), M.p. $87^{\circ} \mathrm{C}-88^{\circ} \mathrm{C}$; IR $v_{\max }(\mathrm{KBr}): 2951,1763,1734,1626$, 1571, 741,693 $\mathrm{cm}^{-1} ;{ }^{1} \mathrm{H}$ NMR $\left(\mathrm{CDCl}_{3}, 400 \mathrm{MHz}\right): \delta 8.37$ $(\mathrm{s}, 1 \mathrm{H}), 8.02(\mathrm{~d}, J=7.14 \mathrm{~Hz}, 2 \mathrm{H}), 7.56-7.47(\mathrm{~m}, 3 \mathrm{H})$, $2.22(\mathrm{~s}, 4 \mathrm{H}), 1.89$ (s, 4H); ${ }^{13} \mathrm{CNMR}\left(\mathrm{CDCl}_{3}, 100 \mathrm{MHz}\right)$ : $\delta 163.86,160.48,157.65,133.65,133.49,131.68,128.76$, $115.71,113.75,38.54,23.28$.

5-(4-Hydroxybenzylidene)-2,2-butylidene-1,3-dioxane4,6-dione (Compound 3b, Table 1): White solid (yield: $88 \%$ ), M.p. $170^{\circ} \mathrm{C}-172^{\circ} \mathrm{C}$; IR $v_{\max }(\mathrm{KBr}): 2965,2957$, 1750, 1700, 1638, 1537, 1400, 1170, $800 \mathrm{~cm}^{-1}$; ${ }^{1} \mathrm{H}$ NMR $\left(\mathrm{CDCl}_{3}, 400 \mathrm{MHz}\right): \delta 8.35(\mathrm{~s}, 1 \mathrm{H}), 8.12(\mathrm{~d}, J=8.82 \mathrm{~Hz}$, 2H), 6.94 (d, $J=8.82 \mathrm{~Hz}, 2 \mathrm{H}), 2.24-2.19$ (m, 4H), 1.90 - $1.85(\mathrm{~m}, 4 \mathrm{H}) ;{ }^{13} \mathrm{CNMR}\left(\mathrm{CDCl}_{3}, 100 \mathrm{MHz},\right): \delta 164.93$, $162.17,158.37,137.88,124.43,116.21,113.67,110.94$, $38.41,23.26$.

5-(4-Methoxybenzylidene)-2,2-butylidene-1,3-dioxane4,6-dione (Compound 3c, Table 1): White solid (yield: $89 \%$ ), M.p. $83^{\circ} \mathrm{C}-85^{\circ} \mathrm{C}$; IR $v_{\max }(\mathrm{KBr}): 2972,2901$, $1756,1718,1624,1573,1455,1381,1183,800 \mathrm{~cm}^{-1} ;{ }^{1} \mathrm{H}$ NMR $\left(\mathrm{CDCl}_{3}, 400 \mathrm{MHz}\right): \delta 8.32(\mathrm{~s}, 1 \mathrm{H}), 8.19(\mathrm{~d}, J=8.97$ $\mathrm{Hz}, 2 \mathrm{H}), 6.98$ (d, $J=8.97 \mathrm{~Hz}, 2 \mathrm{H}), 3.90$ (s, 3H), 2.23 $2.18(\mathrm{~m}, 4 \mathrm{H}), 1.90-1.85(\mathrm{~m}, 4 \mathrm{H}) ;{ }^{13} \mathrm{CNMR}\left(\mathrm{CDCl}_{3}, 100\right.$ $\mathrm{MHz},): \delta 164.62,161.19,157.56,137.48,124.71,114.38$, 113.37, 111.65, 55.68, 38.41, 23.26.

5-(4-Nitrobenzylidene)-2,2-butylidene-1,3-dioxane-4,6dione (Compound 3d, Table 1): Pale yellow solid (yield: $93 \%$ ), M.p. $150^{\circ} \mathrm{C}-152^{\circ} \mathrm{C}$; IR $v_{\max }(\mathrm{KBr}): 2976,2901$, 1763, 1731, 1626, 1605, 1524, 1350, $800 \mathrm{~cm}^{-1}$; ${ }^{1} \mathrm{H}$ NMR $\left(\mathrm{CDCl}_{3}, 400 \mathrm{MHz}\right): \delta 8.41(\mathrm{~s}, 1 \mathrm{H}), 8.30(\mathrm{~d}, J=8.82 \mathrm{~Hz}$, $2 \mathrm{H}), 8.06(\mathrm{~d}, J=8.70 \mathrm{~Hz}, 2 \mathrm{H}), 2.27-2.23(\mathrm{~m}, 4 \mathrm{H}), 1.95$ - $1.90(\mathrm{~m}, 4 \mathrm{H}) ;{ }^{13} \mathrm{CNMR}\left(\mathrm{CDCl}_{3}, 100 \mathrm{MHz}\right): \delta$ 162.36, 159.36, 153.67, 149.26, 137.11, 132.73, 123.30, 119.17, $114.08,36.43,23.00$.

5-(2-Nitrobenzylidene)-2,2-butylidene-1,3-dioxane-4,6dione (Compound 3e, Table 1): Pale yellow solid (yield: 91\%), M.p. $112^{\circ} \mathrm{C}-114^{\circ} \mathrm{C}$; IR $v_{\max }(\mathrm{KBr}): 2963,2908$, $1765,1736,1625,1604,1524,1363,800 \mathrm{~cm}^{-1}$; ${ }^{1} \mathrm{H}$ NMR $\left(\mathrm{CDCl}_{3}, 400 \mathrm{MHz}\right): \delta 8.75(\mathrm{~s}, 1 \mathrm{H}), 8.28(\mathrm{~d}, J=8.22 \mathrm{~Hz}$, $1 \mathrm{H}), 7.78$ - 7.62 (m, 2H), 7.49 (d, $J=7.56 \mathrm{~Hz}, 1 \mathrm{H}), 2.24$
- $2.21(\mathrm{~m}, 4 \mathrm{H}), 1.91-1.89(\mathrm{~m}, 4 \mathrm{H}) ;{ }^{13} \mathrm{CNMR}\left(\mathrm{CDCl}_{3}\right.$, $100 \mathrm{MHz}): \delta 161.69,159.22,155.09,145.99,133.46$, $130.57,129.99,129.39,124.44,118.19,114.14,38.23$, 22.87 .

5-(4-Furylmethylene)-2,2-butylidene-1,3-dioxane-4,6dione (Compound 3e, Table 1): White solid (yield: 87\%), M.p. $86^{\circ} \mathrm{C}-88^{\circ} \mathrm{C}$; IR $v_{\max }(\mathrm{KBr}): 2961,2943,1757,1726$, 1598, 1604, 1508, 1364, 1081, 840, $804 \mathrm{~cm}^{-1}$; ${ }^{1} \mathrm{H}$ NMR $\left(\mathrm{CDCl}_{3}, 400 \mathrm{MHz}\right): \delta 8.33(\mathrm{~s}, 1 \mathrm{H}), 8.14(\mathrm{~d}, J=5.58,8.64$ $\mathrm{Hz}, 2 \mathrm{H}), 7.16$ (t, $J=8.61 \mathrm{~Hz}, 1 \mathrm{H}), 2.24-2.19$ (m, 4H), $1.91-1.86(\mathrm{~m}, 4 \mathrm{H}) ;{ }^{13} \mathrm{CNMR}\left(\mathrm{CDCl}_{3}, 100 \mathrm{MHz},\right): \delta$ $167.44,164.01,163.81,160.54,156.21,136.73,136.60$, $128.07,128.03,116.27,115.98,114.99,114.96,113.74$, $38.49,23.24$.

\section{Acknowledgements}

The authors thank the financial support from the National Science and Technology Project (No. 2001BA323C) and the Graduate Innovation Foundation of Jiangxi Province (No.YC10A051).

\section{REFERENCES}

[1] R. T. Acobs, A. D. Wright and F. X. Smith, "Condensation of Monosubstituted Isopropylidene Malonates with Mannich Bases," Journal of Organic Chemistry, Vol. 47, No. 19, 1982, pp. 3769-3772.

http://dx.doi.org/10.1021/jo00140a038

[2] R. Bruns, A. Wernicke and P. Koll, "Application of the Reaction of D-Glucose with Meldrum's Acid: Total Synthesis of the Styryl Lactones (+)-Goniofufurone and (+)7-epi-goniofufurone," Tetrahedron, Vol. 55, No. 32, 1999, pp. 9793-9800.

[3] I. N. Nestrova, A. K. Shanazarov and A. M. Poznyak, "Improved Method of Synthesis 2,2-Dimethyl-4,6-dioxo1,3-dioxane (Meldrum's Acid)," Pharmaceutical Chemistry Journal, Vol. 28, No. 8, 1994, pp. 583-585. http://dx.doi.org/10.1007/BF02219035

[4] E. M. Abdelaziz, A. C. Jhonny Azuaje, C. Ernesto, Y. C. Matilde, Y. C. Vicente and S. Eddy, "Discovery and Preliminary SAR of 5-Arylidene-2,2-dimethyl-1,3-dioxane-4, 6-diones as Platelet Aggregation Inhibitors," Combinatorial Chemistry \& High Throughput Screening, Vol. 15, No. 7, 2012, pp. 551-554. http://dx.doi.org/10.2174/138620712801619122

[5] W. G. Rajecwarna, R. B. Labroo and L. A. Cohen, "Synthesis of 5-[(Indol-2-on-3-yl)methyl]-2,2-dimethyl-1,3-dioxane-4,6-diones and Spirocyclopropyloxindole Derivatives. Potential Aldose Reductase Inhibitors," Journal of Organic Chemistry, Vol. 64, No. 4, 1999, pp. 1369-1371.

[6] T. Watanabe, T. F. Knpfel and E. M. Carreira, "Asymmetric Conjugate Addition Reactions of Meldrum's Acid Derived Acceptors Employing Chiral Phosphoramidite Ligands," Organic Letters, Vol. 5, No. 25, 2003, pp. 4557 4558. http://dx.doi.org/10.1021/o1035575q

[7] E. Fillion, A. M. Dumas and S. A. Hogg, "Modular, 
Modular Synthesis of Tetrahydrofluorenones from 5-Alkylidene Meldrum's Acids," The Journal of Organic Chemistry, Vol. 71, No. 26, 2006, pp. 9899-9902. http://dx.doi.org/10.1021/jo0618876

[8] S. Rádl, J. Stach and J. Hajicek, "An Improved Synthesis of 1,1-Dimethylethyl6-cyanomethyl-2,2-dimethyl-1,3-dioxane-4-acetate, a Key Intermediate for Atorvastatin Synthesis," Tetrahedron Letters, Vol. 43, No. 11, 2002, pp. 2087-2090.

[9] D. Davidson and S. A. Bernhard, "The Structure of Meldrum's Supposed $\beta$-Lactonic Acid," Journal of the American Chemical Society, Vol. 70, No. 10, 1948, pp. 34263428. http://dx.doi.org/10.1021/ja01190a060

[10] E. J. Corey, "The Mechanism of the Decarboxylation of $\alpha, \beta$ - and $\beta, \gamma$-Unsaturated Malonic Acid Derivatives and the Course of Decarboxylative Condensation Reactions in Pyridine," Journal of the American Chemical Society, Vol. 74, 1952, pp. 5897-5905. http://dx.doi.org/10.1021/ja01143a021

[11] G. A. Kraus and M. E. Krolski, "Synthesis of a Precursor to Quassimarin," Journal of Organic Chemistry, Vol. 51, No. 17, 1986, pp. 3347-3450. http://dx.doi.org/10.1021/jo00367a017

[12] P. Scuster, O. E. Polansky and F. Wessely, "Zur Kenntnis cyclischer Acylale, 6. Mitt.," Monatshefte für Chemie, Vol. 95, 1964, pp. 53-58. http://dx.doi.org/10.1007/BF00909251

[13] Z. H. Xu, N. Yan and W. L. Liao, "Knoevenagel Condensation Reaction of 2,2-Dimethyl-1,3-dioxane-4,6-dione and Aromatic Aldehydes by Hexamethyldisilazane," Chinese Journal of Jiangxi Normal University (Natural Science), Vol. 36, No. 5, 2012, pp. 524-526.

[14] P. S. Rao and R. V. Venkataratnam, "Zinc Chloride as a New Catalyst for Knoevenagel Condensation," Tetrahedron Letters, Vol. 32, No. 41, 1991, pp. 5821-5822. http://dx.doi.org/10.1016/S0040-4039(00)93564-0

[15] A. M. Dumas and E. Fillion, "Meldrum's Acids and 5Alkylidene Meldrum's Acids in Catalytic Carbon-Carbon Bond-Forming Processes," Accounts of Chemical Research, Vol. 43, No. 3, 2009, pp. 440-454. http://dx.doi.org/10.1021/ar900229z

[16] Z. H. Xu and C. H. L, "Solvent-Free Synthesis of 5-Alkenyl-2,2-pentamethylene-1,3-dioxane-4,6-diones under U1trasonic Irradiation with $\mathrm{CePW}_{12} \mathrm{O}_{40}$ as Catalyst," Chinese Journal of Organic Chemistry, Vol. 33, No. 7, 2013, pp. 1540-1544.
[17] A. M. Dumas, A. Seed, A. K. Zorzitto and E. Fillion, "A General and Practical Preparation of Alkylidene Meldrum's Acids," Tetrahedron Letters, Vol. 48, No. 40, 2007, pp. 7072-7074.

http://dx.doi.org/10.1016/j.tetlet.2007.08.012

[18] B. Janardhan, G. Rajitha, V. Ravibabu, B. Rajitha and A. C. Peter, "An Eco-Friendly Improved Protocol for the Synthesis of Bis(3-indolyl)methanes Using Poly(4-vinylpyridinium)hydrogen Sulfate as Efficient, Heterogeneous, and Recyclable Solid Acid Catalyst," ISRN Organic Chemistry, Vol. 2013, 2013, pp. 1-5.

[19] Z. H. Jun, J. T. Li and Y. J. Bian, "Pinacolization of Aromatic Aldehydes Using $\mathrm{Zn} / \mathrm{Montmorillonite} \mathrm{K} 10-\mathrm{ZnCl}_{2}$ in Aqueous THF under Ultrasound," Chemical Journal on Internet, Vol. 5, No. 1, 2003, pp. 8-9.

[20] J. T. Li, X. H. Zhang and Z. P. Lin, “An Improved Synthesis of 1,3,5-Triaryl-2-pyrazolines in Acetic Acid Aqueous Solution under Ultrasound Irradiation," Beilstein Journal of Organic Chemistry, Vol. 13, No. 3, 2007, pp. 1-4.

[21] T. P. Caulier and J. Reisse, "On Sonochemical Effects on the Diels-Alder Reaction," Journal of Organic Chemistry, Vol. 60, No. 7, 1996, pp. 2547-2548. http://dx.doi.org/10.1021/jo9520203

[22] M. M. Mojtahedi, M. S. Abaee, M. Samianifard, A. Shamloo, M. Padyab, A. W. Mesbah and K. Harms, "Ultrasound Mediation for Efficient Synthesis of Monoarylidene Derivatives of Homo- and Heterocyclic Ketones," Ultrasonics Sonochemistry, Vol. 20, No. 3, 2013, pp. 924-930. http://dx.doi.org/10.1016/j.ultsonch.2012.11.004

[23] A. Lahyani, M. Chtourou, M. H. Frikha and M. Trabels, "Amberlyst-15 and Amberlite-200C: Efficient Catalysts for Aldol and Cross-Aldol Condensation under Ultrasound Irradiation," Ultrasonics Sonochemistry, Vol. 20, No. 5, 2013, pp. 1296-1303. http://dx.doi.org/10.1016/j.ultsonch.2013.01.017

[24] N. Yan, B. Xiong, W. L. Liao and Z. H. Xu, "Catalytic Synthesis of 1,3-Dioxane-4,6-dione by $\mathrm{La}(\mathrm{OTf})_{3}$, , Chinese Journal of Organic Chemistry, Vol. 30, No. 30, 2010, pp. 1391-1394.

[25] Z. H. Xu, C. H. Lin and J. H. Xia, "An Efficient Synthesis of 1,3-Dioxane-4,6-diones by Boric Acid," Heterocyclic Letters, Vol. 3, No. 3, 2013, pp. 319-323.

[26] F. P. Xu, W. F. Ding and B. L. Da, "Reactions of 2,2Pentamethylene-1,3-dioxan-4,6-dione with Triethyl Orthoformate and Aromatic Amines," Chinese Journal of Organic Chemistry, Vol. 11, No. 3, 1991, pp. 318-321. 